\title{
Contrastes entre procedências de híbridos de milho em relação à suscetibilidade aos herbicidas nicosulfuron e isoxaflutole
}

\author{
Contrasts among origins of corn hybrids in relation to their \\ susceptibility to nicosulfuron and isoxaflutole herbicides
}

\author{
Sidnei Douglas Cavalieri ${ }^{*}$; Rubem Silvério de Oliveira Junior ${ }^{2}$; \\ Jamil Constantin ${ }^{2}$; Denis Fernando Biffe ${ }^{3}$; Diego Gonçalves Alonso ${ }^{3}$; \\ João Guilherme Zanetti de Arantes ${ }^{3}$; Rosecler Salete Canossa ${ }^{4}$
}

\section{Resumo}

\begin{abstract}
A seletividade de herbicidas é a base para o sucesso do controle químico de plantas daninhas na produção agrícola, sendo considerada uma medida da resposta diferencial de diversas espécies de plantas a um determinado herbicida. Objetivou-se neste trabalho avaliar o efeito da procedência de híbridos de milho em relação à suscetibilidade aos herbicidas nicosulfuron e isoxaflutole. $\mathrm{O}$ estudo envolveu dois experimentos, um com nicosulfuron no período de 09/09/05 a 24/10/05 e outro com isoxaflutole no período de 09/10/04 a 10/11/04, ambos realizados em casa-de-vegetação. Os experimentos foram conduzidos no delineamento de blocos ao acaso, em arranjo fatorial de $33 \times 3$ para o nicosulfuron e $23 \times 3$ para o isoxaflutole, com quatro repetições, sendo que o primeiro fator constituído por híbridos de milho e o segundo por dosagens dos herbicidas. Após a aplicação do herbicida, avaliou-se a massa seca de parte aérea das plantas. Existem diferenças de tolerância entre procedências de híbridos de milho em relação à suscetibilidade aos herbicidas nicosulfuron e isoxaflutole. Constatou-se que, em média, as procedências Balu e Coodetec foram mais sensíveis ao nicosulfuron do que as demais procedências. Na dosagem de $120 \mathrm{~g} \mathrm{ha}^{-1}$ de isoxaflutole, em média, a procedência Balu mostrou-se mais tolerante que a Embrapa.
\end{abstract}

Palavras-chave: Seletividade, tolerância, sulfoniluréias, isoxazoles

\begin{abstract}
Herbicide selectivity is the basis for successfull weed chemical control in agricultural production, as it is considered as a measurement of the differential response of different plant species to a certain herbicide. The objective of this work was to evaluate the effect of the origin of corn hybrid in relation to its susceptibility to the herbicides nicosulfuron and isoxaflutole. The research was conducted in greenhouse conditions and comprised two experiments, being the first one with nicosulfuron (09/09/2005 to $10 / 24 / 2005)$ and another one with isoxaflutole (10/09/04 to 11/10/04). Both experiments were run in a randomized blocks design, in a factorial scheme $33 \times 3$ for nicosulfuron and $23 \times 3$ for isoxaflutole, with four replicates. The first factor was constituted by corn hybrids and the second one by herbicide rates.
\end{abstract}

\footnotetext{
${ }^{1}$ Eng $^{\circ}$-Agro ${ }^{\circ}$ D.Sc. Pesquisador da Embrapa Hortaliças - Centro Nacional de Pesquisa de Hortaliças (CNPH). E-mail: cavalieri@cnph.embrapa.br

${ }^{2}$ Eng $^{\circ}$-Agr ${ }^{\circ}$, D.Sc. Professor Associado, Coordenador do Núcleo de Estudos Avançados em Ciência das Plantas Daninhas, NAPD/UEM, Departamento de Agronomia, Universidade Estadual de Maringá, UEM. Bolsista CNPq. E-mail: rsojunior@, uem.br; constant@teracom.com.br

${ }^{3}$ Eng $^{\circ}$-Agr ${ }^{\circ}$, M.Sc., Doutorando do Programa de Pós-graduação em Agronomia (Proteção de Plantas), NAPD/UEM, Bolsista Capes e CNPq. E-mail: biffeagro@hotmail.com; alonsodg07@hotmail.com; jgza2004@hotmail.com

${ }^{4}$ Professora Visitante da Faculdade de Sinop, FASIP. E-mail: r_canossa@hotmail.com

* Autor para correspondência
} 
After herbicide spraying, shoot dry biomass was evaluated. It was concluded that origins of corn hybrids affects the susceptibility to the herbicides nicosulfuron and isoxaflutole. In average, Balu and Codetec hybrids were the most sensitive to nicosulfuron. For isoxaflutole applied at $120 \mathrm{~g} \mathrm{ha}^{-1}$ Balu hybrids were more tolerant than Embrapa hybrids.

Key words: Selectivity, tolerance, sulfonylureas, isoxasoles

\section{Introdução}

O nicosulfuron é um herbicida sistêmico pertencente ao grupo químico das sulfoniluréias, que se destaca entre os principais pós-emergentes utilizados na cultura do milho, principalmente para o controle de gramíneas e algumas dicotiledôneas anuais (RODRIGUES; ALMEIDA, 2005). Os herbicidas deste grupo inibem a acetolactato sintase (ALS), a primeira enzima comum à rota de biossíntese dos aminoácidos de cadeia ramificada, valina, leucina e isoleucina, em plantas e microrganismos (ANDERSON et al., 1998).

A seletividade dos herbicidas do grupo das sulfoniluréias para as culturas baseia-se nas diferentes taxas de metabolização dos mesmos pelas plantas (CAREY; PENNER; KELLS, 1997) e na velocidade de absorção e translocação. Espécies tolerantes detoxificam rapidamente estes herbicidas, transformando-os em compostos não fitotóxicos pela ação do citocromo P450 monoxigenase, em reações de hidroxilação e glioxilação (FONNEPFISTER et al., 1990). O principal mecanismo das plantas na metabolização de sulfoniluréias parece ser a hidroxilação, que freqüentemente resulta em detoxificação do herbicida (HARMS et al., 1990). No entanto, em alguns casos, reações de glicosilação são também requeridas para completar a detoxificação (BROW et al., 1991).

A tolerância dos híbridos de milho aos herbicidas pós-emergentes do grupo das sulfoniluréias é bastante variável, podendo ser elevada para alguns e reduzida para outros. Híbridos considerados tolerantes a estes herbicidas podem apresentar sensibilidade diferenciada, dependendo do estádio de desenvolvimento da planta, do ambiente e da dosagem utilizada (GUBBIGA; WORSHAM; COBLE, 1995).
$\mathrm{O}$ isoxaflutole pertence ao grupo químico dos isoxasoles, sendo considerado um pró-herbicida, uma vez que no solo, água e planta é rapidamente convertido ao metabólito diquetonitrila, que é a molécula biologicamente ativa no controle de plantas daninhas (CEZARINO, 1997).

A grande responsável pela seletividade do isoxaflutole em espécies tolerantes, tais como o milho e a cana-de-açúcar, é a capacidade de metabolizar rapidamente o isoxaflutole para diquetonitrila e, principalmente, deste para ácido benzóico, que não possui ação herbicida, e, ao final do processo, para gás carbônico (PALLET et al., 1998; SPRAGUE; PENNER; KELLS, 1999). Em espécies sensíveis, essa metabolização ocorre lentamente, permitindo a inibição enzimática pelo diquetonitrila. Parece não haver diferenças na absorção e translocação entre milho e plantas sensíveis, com o diquetonitrila sendo translocado através de toda a planta, para folhas novas, predominantemente pela rota apoplástica (PALLET et al., 1998).

O isoxaflutole causa a disrupção da síntese de pigmentos, levando ao surgimento de sintomas característicos de "branqueamento" de tecidos desenvolvidos após a aplicação nas espécies suscetíveis, seguido de paralisação de crescimento e necrose (PALLET et al., 1998).

A maioria dos herbicidas inibidores da síntese de carotenóides, no entanto, atua sobre a fitoeno desaturase, enzima que cataliza as reações iniciais na conversão do fitoeno (precursor incolor dos carotenóides) em carotenóides coloridos. Os sintomas foliares estão associados com o acúmulo do fitoeno, típico dos herbicidas que inibem o fitoeno desaturase. No entanto, nem o isoxaflutole nem o diquetonitrila inibem a atividade desta enzima. O diquetonitrila inibe a 4-hidroxifenil- 
piruvato dioxigenase (HPPD) a partir da degradação do aminoácido tirosina, com inibição indireta do fitoeno desaturase resultante da depleção de um co-fator essencial, a plastoquinona (PALLET et al., 1998; VIVIANI; LITTLE; PALLET, 1998).

Em decorrência da fitointoxicação apresentada pela cultura do milho após a aplicação dos herbicidas nicosulfuron e isoxaflutole, objetivou-se neste trabalho avaliar o efeito da procedência dos híbridos de milho em relação à suscetibilidade a estes herbicidas.

\section{Material e Métodos}

O presente trabalho foi conduzido em casa-devegetação do Núcleo de Estudos Avançados em Ciência das Plantas Daninhas da Universidade
Estadual de Maringá (NAPD/UEM), no município de Maringá, PR (2325’31'S, 5156’19”O), a 542 $\mathrm{m}$ de altitude. Segundo a classificação de Köppen, o clima da localidade é do tipo CW'a, mesotérmico úmido, com chuvas de verão e de outono e verão quente.

Foram conduzidos dois experimentos, um com o herbicida nicosulfuron no período de 09/09/05 a $24 / 10 / 05$ e outro com o isoxaflutole no período de 09/10/04 a 10/11/04, utilizando-se amostras deformadas de solo (ARGISSOLO VERMELHO DISTRÓFICO), segundo Empresa Brasileira de Pesquisa Agropecuária (1999) provenientes da camada de 0 a $0,20 \mathrm{~m}$ de profundidade. As características químicas e granulométrica dos solos utilizados em cada experimento, respectivamente, encontram-se nas Tabelas 1 e 2.

Tabela 1. Resultado das análises químicas e granulométrica do solo utilizado no experimento (camada de 0 a $20 \mathrm{~cm}$ de profundidade). Maringá-PR, 2005.

\begin{tabular}{|c|c|c|c|c|c|c|c|c|c|c|}
\hline \multicolumn{2}{|c|}{$\mathbf{p H}$} & $\mathbf{A l}^{3+}$ & $\mathbf{H}^{+}+\mathbf{A l}^{3+}$ & $\mathrm{Ca}^{2+}$ & $\mathbf{M g}^{2+}$ & $\mathbf{K}^{+}$ & SB & CTC & \multirow{2}{*}{$\frac{\mathbf{P}}{\left(\mathrm{mg} \mathrm{dm}^{-3}\right)}$} & \multirow{2}{*}{$\frac{\mathbf{C}}{\left(\mathrm{g} \mathrm{dm}^{-3}\right)}$} \\
\hline $\mathrm{CaCl}_{2}$ & $\mathrm{H}_{2} \mathrm{O}$ & \multicolumn{7}{|c|}{$\left(\mathrm{cmol}_{\mathrm{c}} \mathrm{dm}^{-3}\right)$} & & \\
\hline 5,6 & 6,4 & 0,0 & 2,54 & 2,88 & 0,62 & 0,38 & 3,88 & 6,42 & 104,0 & 7,11 \\
\hline \multicolumn{3}{|c|}{ Areia grossa } & \multicolumn{3}{|c|}{ Areia fina } & \multicolumn{3}{|c|}{ Silte } & \multicolumn{2}{|c|}{ Argila } \\
\hline \multicolumn{11}{|c|}{$\left(\mathrm{g} \mathrm{kg}^{-1}\right)$} \\
\hline \multicolumn{3}{|c|}{430} & \multicolumn{3}{|c|}{370} & \multicolumn{2}{|c|}{60} & & \multicolumn{2}{|c|}{140} \\
\hline
\end{tabular}

Fonte: Laboratório de Solos da Universidade Estadual de Maringá (UEM).

Tabela 2. Resultado das análises químicas e granulométrica do solo utilizado no experimento (camada de 0 a $20 \mathrm{~cm}$ de profundidade). Maringá-PR, 2004.

\begin{tabular}{|c|c|c|c|c|c|c|c|c|c|c|}
\hline \multicolumn{2}{|c|}{ pH } & $\mathbf{A} \mathbf{l}^{3+}$ & $\mathbf{H}^{+}+\mathrm{Al}^{3+}$ & $\mathrm{Ca}^{2+}$ & $\mathbf{M g}^{2+}$ & $\mathbf{K}^{+}$ & SB & CTC & $\mathbf{P}$ & C \\
\hline $\mathrm{CaCl}_{2}$ & $\mathrm{H}_{2} \mathrm{O}$ & & & & $\mathrm{nol}_{\mathrm{c}} \mathrm{dn}$ & & & & $\left(\mathrm{mg} \mathrm{dm}^{-3}\right)$ & $\left(\mathrm{g} \mathrm{dm}^{-3}\right)$ \\
\hline 5,6 & 6,5 & 0,0 & 1,88 & 1,48 & 0,59 & 0,16 & 2,23 & 4,11 & 51 & 5,58 \\
\hline \multicolumn{3}{|c|}{ Areia grossa } & \multicolumn{2}{|c|}{ Areia fina } & & \multicolumn{2}{|c|}{ Silte } & & \multicolumn{2}{|c|}{ Argila } \\
\hline \multicolumn{11}{|c|}{$\left(\mathrm{g} \mathrm{kg}^{-1}\right)$} \\
\hline \multicolumn{3}{|c|}{650} & \multicolumn{2}{|c|}{300} & & \multicolumn{2}{|c|}{20} & & \multicolumn{2}{|c|}{30} \\
\hline
\end{tabular}

Fonte: Laboratório de Solos da Universidade Estadual de Maringá (UEM). 
As amostras foram coletadas em área com conhecido histórico de uso sob semeadura direta. Após a coleta das amostras, o solo foi peneirado em malha de $2 \mathrm{~mm}$ para separação de torrões, raízes e palha.

Em ambos os experimentos as unidades experimentais foram constituídas por vasos com capacidade de $5 \mathrm{dm}^{3}$, onde foram semeadas 6 sementes de milho por vaso, à profundidade de aproximadamente $2 \mathrm{~cm}$. A semeadura foi efetuada com sementes tratadas com imidacloprid na dosagem de 480 g para cada $100 \mathrm{~kg}$ de sementes. Após a emergência das plantas, procedeu-se o desbaste, deixando apenas duas e três plantas por vaso, para os experimentos em que foram aplicados nicosulfuron e isoxaflutole, respectivamente.

A aplicação do nicosulfuron foi realizada quando as plantas de milho apresentavam-se com 6 folhas totalmente expandidas e para isoxaflutole foi realizada em pré-emergência, logo após a semeadura. As aplicações foram realizadas com pulverizador costal pressurizado com $\mathrm{CO}_{2}$, com pressão constante de $207 \mathrm{kPa}$, equipado com três pontas XR 110.02, espaçadas de $0,5 \mathrm{~m}$ entre si e posicionadas a $0,5 \mathrm{~m}$ da superfície do alvo, utilizando-se o equivalente a $200 \mathrm{~L} \mathrm{ha}^{-1}$ de calda.

No experimento com nicosulfuron foram avaliados 33 híbridos de milho (2A 525, 2B 170, 2C 599, CO 32, P 30F33, P 30F90, P 30F98, A 010, A 015, A 2555, AG 7000, AG 8081, AG 8060, AG 9090, AS 1548, AS 1565, AS 1567, AS 1570, AS 1575, B 184, B 551, B 761, BRS 3003, BRS 3150, CD 304, CD 308, Ocepar 705, Fort, Garra,
Maximus, Penta, Tork e SG 6418) oriundos de nove procedências (Agroceres, Agroeste, Balu, Dow, Embrapa, Guerra, Nidera, Pionner, Syngenta). Para o isoxaflutole foram avaliados 23 híbridos (AG 6040, AG 9010, B 178, B 184, B 551, B 761, BRS 2114, BRS 2160, BRS 3003, BRS 3150, CD 304, CD 306, CD 307, CD 308, Ocepar 705, Fort, Garra, Penta, Pointer, Premium-Flex, Speed, Tork e Valente), das mesmas procedências.

Em ambos os experimentos os híbridos foram combinados em esquema fatorial com três dosagens dos herbicidas nicosulfuron $\left(0,30\right.$ e $\left.60 \mathrm{~g} \mathrm{ha}^{-1}\right)$ e isoxaflutole $\left(0,60\right.$ e $\left.120 \mathrm{~g} \mathrm{ha}^{-1}\right)$, no delineamento de blocos ao acaso com 4 repetições.

Avaliou-se a massa seca de parte aérea das plantas de cada vaso aos 14 e 32 DAA para nicosulfuron e isoxaflutole, respectivamente. Tal avaliação foi realizada mediante a colheita do material vegetal presente nos vasos, com posterior secagem em estufa a $70^{\circ} \mathrm{C}$ por 48 horas. A avaliação de massa seca foi corrigida para valores percentuais por meio da comparação dos valores obtidos nos tratamentos herbicidas com os valores da testemunha (dosagem de $\left.0 \mathrm{~g} \mathrm{ha}^{-1}\right)$, considerada $100 \%$.

Os dados foram submetidos aos testes de Levene e Shapiro-Wilk, com o objetivo de avaliar variância e normalidade dos erros, utilizando-se o programa estatístico SAS (STATISTICAL ANALYSIS SYSTEM, 1999). Posteriormente foram submetidos à análise de variância, sendo submetidos a contrastes (Tabelas 3 e 4) previamente estabelecidos pelo teste F a 5\% de probabilidade, utilizando-se o programa estatístico SISVAR (FERREIRA, 1999). 
Tabela 3. Contrastes entre procedências dos híbridos avaliados no experimento com nicosulfuron. Maringá-PR, 2005.

\begin{tabular}{|c|c|c|c|c|c|c|}
\hline \multirow{2}{*}{\multicolumn{4}{|c|}{ Contrastes }} & \multicolumn{3}{|c|}{ Componentes de cada grupo nos contrastes } \\
\hline & & & & \multicolumn{3}{|c|}{ Híbridos } \\
\hline$\hat{C}_{1}$ & Agroceceres & $v s$ & Agroeste & AG 7000, AG 8021, AG 8060 e AG 9090 & $v s$ & $\begin{array}{l}\text { AS } 1548, \text { AS } 1565, \text { AS } 1567, \\
\text { AS } 1570 \text { e AS } 1575\end{array}$ \\
\hline$\hat{C}_{2}$ & Agroceceres & $v s$ & Balu & AG 7000, AG 8021, AG 8060 e AG 9090 & $v s$ & B 184, B 551 e B 761 \\
\hline$\hat{C}_{3}$ & Agroceceres & vs & Coodetec & AG 7000, AG 8021, AG 8060 e AG 9090 & $v s$ & $\begin{array}{l}\text { CD 304, CD } 308 \text { e Ocepar } \\
705\end{array}$ \\
\hline$\hat{C}_{4}$ & Agroceceres & $v s$ & Dow & AG 7000, AG 8021, AG 8060 e AG 9090 & $v s$ & $\begin{array}{c}2 \mathrm{~A} 525,2 \mathrm{~B} 170,2 \mathrm{C} 599 \mathrm{e} \\
\text { CO } 32\end{array}$ \\
\hline$\hat{C}_{5}$ & Agroceceres & $v s$ & Embrapa & AG 7000, AG 8021, AG 8060 e AG 9090 & $v s$ & BRS 3003 e BRS 3150 \\
\hline$\hat{C}_{6}$ & Agroceceres & $v s$ & Guerra & AG 7000, AG 8021, AG 8060 e AG 9090 & $v s$ & SG 6418 \\
\hline$\hat{C}_{7}$ & Agroceceres & $v s$ & Nidera & AG 7000, AG 8021, AG 8060 e AG 9090 & $v s$ & A 010, A 015 e A 2555 \\
\hline$\hat{C}$ & Agroceceres & $v s$ & Pionner & AG 7000, AG 8021, AG 8060 e AG 9090 & $v s$ & P 30F33, P 30F90 e P 30F98 \\
\hline$\hat{C}_{9}$ & Agroceceres & $v s$ & Syngenta & AG 7000, AG 8021, AG 8060 e AG 9090 & $v s$ & $\begin{array}{c}\text { Fort, Garra, Maximus, Penta } \\
\text { e Tork }\end{array}$ \\
\hline$\hat{C}_{10}$ & Agroeste & $v s$ & Balu & $\begin{array}{c}\text { AS 1548, AS 1565, AS1567, AS } 1570 \text { e } \\
\text { AS } 1575\end{array}$ & $v s$ & B 184, B551 e B 761 \\
\hline$\hat{C}_{11}$ & Agroeste & $v s$ & Coodetec & $\begin{array}{c}\text { AS 1548, AS 1565, AS1567, AS } 1570 \text { e } \\
\text { AS } 1575\end{array}$ & $v s$ & $\begin{array}{l}\text { CD 304, CD } 308 \text { e Ocepar } \\
705\end{array}$ \\
\hline$\hat{C}_{12}$ & Agroeste & $v s$ & Dow & $\begin{array}{c}\text { AS } 1548, \text { AS } 1565, \text { AS } 1567, \text { AS } 1570 \text { e } \\
\text { AS } 1575\end{array}$ & $v s$ & $\begin{array}{c}2 \mathrm{~A} 525,2 \mathrm{~B} 170,2 \mathrm{C} 599 \mathrm{e} \\
\text { CO } 32\end{array}$ \\
\hline$\hat{C}_{13}$ & Agroeste & $v s$ & Embrapa & $\begin{array}{c}\text { AS } 1548, \text { AS } 1565 \text {, AS1567, AS } 1570 \text { e } \\
\text { AS } 1575\end{array}$ & $v s$ & BRS 3003 e BRS 3150 \\
\hline$\hat{C}_{14}$ & Agroeste & $v s$ & Guerra & $\begin{array}{c}\text { AS } 1548, \text { AS } 1565, \text { AS } 1567, \text { AS } 1570 \text { e } \\
\text { AS } 1575\end{array}$ & $v s$ & SG 6418 \\
\hline$\hat{C}_{15}$ & Agroeste & $v s$ & Nidera & $\begin{array}{c}\text { AS 1548, AS 1565, AS1567, AS } 1570 \text { e } \\
\text { AS } 1575\end{array}$ & $v s$ & A 010, A 015 e A 2555 \\
\hline$\hat{C}_{16}$ & Agroeste & $v s$ & Pionner & $\begin{array}{c}\text { AS 1548, AS 1565, AS1567, AS } 1570 \text { e } \\
\text { AS } 1575\end{array}$ & $v s$ & P 30F33, P 30F90 e P $30 F 98$ \\
\hline$\hat{C}_{17}$ & Agroeste & $v s$ & Syngenta & $\begin{array}{c}\text { AS 1548, AS 1565, AS1567, AS } 1570 \text { e } \\
\text { AS } 1575\end{array}$ & $v s$ & $\begin{array}{c}\text { Fort, Garra, Maximus, Penta } \\
\text { e Tork }\end{array}$ \\
\hline$\hat{C}_{18}$ & Balu & $v s$ & Coodetec & B 184, B551 e B 761 & $v s$ & $\begin{array}{c}\text { CD 304, CD } 308 \text { e Ocepar } \\
705\end{array}$ \\
\hline$\hat{C}_{19}$ & Balu & $v s$ & Dow & B 184, B551 e B 761 & $v s$ & $\begin{array}{c}2 \mathrm{~A} 525,2 \mathrm{~B} 170,2 \mathrm{C} 599 \mathrm{e} \\
\text { CO } 32\end{array}$ \\
\hline$\hat{C}_{20}$ & Balu & $v S$ & Embrapa & B 184, B551 e B 761 & $v s$ & BRS 3003 e BRS 3150 \\
\hline$\hat{C}_{21}$ & Balu & $v s$ & Guerra & B 184, B551 e B 761 & $v s$ & SG 6418 \\
\hline$\hat{C}_{22}$ & Balu & $v s$ & Nidera & B 184, B551 e B 761 & $v s$ & A 010 , A 015 e A 2555 \\
\hline$\hat{C}_{23}$ & Balu & $v s$ & Pionner & B 184, B551 e B 761 & $v s$ & P 30F33, P 30F90 e P 30F98 \\
\hline$\hat{C}_{24}$ & Balu & $v s$ & Syngenta & B 184, B551 e B 761 & $v s$ & $\begin{array}{c}\text { Fort, Garra, Maximus, Penta } \\
\text { e Tork }\end{array}$ \\
\hline$\hat{C}_{25}$ & Coodetec & $v s$ & Dow & CD 304, CD 308 e Ocepar 705 & $v s$ & $\begin{array}{c}2 \mathrm{~A} 525,2 \mathrm{~B} 170,2 \mathrm{C} 599 \mathrm{e} \\
\text { CO } 32\end{array}$ \\
\hline
\end{tabular}




\begin{tabular}{|c|c|c|c|c|c|c|}
\hline$\hat{C}_{26}$ & Coodetec & $v s$ & Embrapa & CD 304, CD 308 e Ocepar 705 & $v s$ & BRS 3003 e BRS 3150 \\
\hline$\hat{C}_{27}$ & Coodetec & $v s$ & Guerra & CD 304, CD 308 e Ocepar 705 & $v s$ & SG 6418 \\
\hline & Coodetec & vs & Nidera & CD 304, CD 308 e Ocepar 705 & $v s$ & A 010, A 015 e A 2555 \\
\hline$\hat{C}_{29}$ & Coodetec & $v s$ & Pionner & CD 304, CD 308 e Ocepar 705 & $v s$ & P 30F33, P 30F90 e P 30F98 \\
\hline$\hat{C}$ & Coodetec & vs & Syngenta & CD 304, CD 308 e Ocepar 705 & vs & $\begin{array}{c}\text { Fort, Garra, Maximus, Penta } \\
\text { e Tork }\end{array}$ \\
\hline & Dow & vs & Embrapa & $2 \mathrm{~A} 525,2 \mathrm{~B} 170,2 \mathrm{C} 599$ e CO 32 & vs & BRS 3003 e BRS 3150 \\
\hline$\hat{C}$ & Dow & vs & Guerra & $2 \mathrm{~A} 525,2 \mathrm{~B} 170,2 \mathrm{C} 599$ е CO 32 & vs & SG 6418 \\
\hline$\hat{C}$ & Dow & vs & Nidera & 2A 525,2 B 170,2 C 599 e CO 32 & vs & A 010, A 015 e A 2555 \\
\hline$\hat{\widehat{C}}$ & Dow & vs & Pionner & $2 \mathrm{~A} 525,2 \mathrm{~B} 170,2 \mathrm{C} 599$ е CO 32 & vs & P 30F33, P 30F90 e P 30F98 \\
\hline$\hat{C}^{34}$ & Dow & vs & Syngenta & 2A 525,2 B 170,2 C 599 e CO 32 & vs & $\begin{array}{c}\text { Fort, Garra, Maximus, Penta } \\
\text { e Tork }\end{array}$ \\
\hline & Embrapa & vs & Guerra & BRS 3003 e BRS 3150 & vs & SG 6418 \\
\hline$\hat{C}$ & Embrapa & vs & Nidera & BRS 3003 e BRS 3150 & vs & A 010 , A 015 e A 2555 \\
\hline$\hat{C}_{38}$ & Embrapa & $v s$ & Pionner & BRS 3003 e BRS 3150 & $v s$ & $30 \mathrm{~F} 33,30 \mathrm{~F} 90$ e $30 \mathrm{~F} 98$ \\
\hline$\hat{C}_{39}$ & Embrapa & $v s$ & Syngenta & BRS 3003 e BRS 3150 & $v s$ & $\begin{array}{c}\text { Fort, Garra, Maximus, Penta } \\
\text { e Tork }\end{array}$ \\
\hline$\hat{C}_{40}$ & Guerra & $v S$ & Nidera & SG 6418 & $v s$ & A 010 , A 015 e A 2555 \\
\hline$\hat{C}_{41}$ & Guerra & $v s$ & Pionner & SG 6418 & $v s$ & P 30F33, P 30F90 e P 30F98 \\
\hline$\hat{C}_{42}$ & Guerra & $v s$ & Syngenta & SG 6418 & $v s$ & $\begin{array}{c}\text { Fort, Garra, Maximus, Penta } \\
\text { e Tork }\end{array}$ \\
\hline$\hat{C}_{43}$ & Nidera & $v S$ & Pionner & A 010, A 015 e A 2555 & $v s$ & 30F33, 30F90 e 30F98 \\
\hline$\hat{C}_{44}$ & Nidera & $v s$ & Syngenta & A 010, A 015 e A 2555 & $v s$ & $\begin{array}{c}\text { Fort, Garra, Maximus, Penta } \\
\text { e Tork }\end{array}$ \\
\hline$\hat{C}_{45}$ & Pionner & $v s$ & Syngenta & $30 \mathrm{~F} 33,30 \mathrm{~F} 90$ e $30 \mathrm{~F} 98$ & & $\begin{array}{c}\text { Fort, Garra, Maximus, Penta } \\
\text { e Tork }\end{array}$ \\
\hline
\end{tabular}

Tabela 4. Contrastes entre procedências dos híbridos avaliados no experimento com isoxaflutole. Maringá-PR, 2004.

\begin{tabular}{|c|c|c|c|c|c|c|}
\hline \multirow{2}{*}{\multicolumn{4}{|c|}{ Contrastes }} & \multicolumn{3}{|c|}{ Componentes de cada grupo nos contrastes } \\
\hline & & & & \multicolumn{3}{|c|}{ Híbridos } \\
\hline$\hat{C}_{1}$ & Agroceceres & vs & Balu & AG 6040 e AG 9010 & vs & B 178, B 184, B551 e B 761 \\
\hline$\hat{C}_{2}$ & Agroceceres & vs & Coodetec & AG 6040 e AG 9010 & vs & $\begin{array}{c}\text { CD 304, CD 306, CD 307, CD } \\
308 \text { e Ocepar } 705\end{array}$ \\
\hline$\hat{C}_{3}$ & Agroceceres & vs & Embrapa & AG 6040 e AG 9010 & vs & $\begin{array}{c}\text { BRS 2114, BRS 2160, BRS } 3003 \\
\text { e BRS } 3150\end{array}$ \\
\hline
\end{tabular}




\begin{tabular}{|c|c|c|c|c|c|c|}
\hline$\hat{C}_{4}$ & Agroceceres & vs & Syngenta & AG 6040 e AG 9010 & vs & $\begin{array}{c}\text { Fort, Garra, Penta, Pointer, } \\
\text { Premium Flex, Speed, Tork e } \\
\text { Valent }\end{array}$ \\
\hline$\hat{C}_{5}$ & Balu & vs & Coodetec & B 178, B 184, B551 e B 761 & vs & $\begin{array}{c}\text { CD 304, CD 306, CD 307, CD } \\
308 \text { e OCEPAR } 705\end{array}$ \\
\hline$\hat{C}_{6}$ & Balu & vs & Embrapa & B 178, B 184, B551 e B 761 & vs & $\begin{array}{c}\text { BRS 2114, BRS 2160, BRS } 3003 \\
\text { e BRS } 3150\end{array}$ \\
\hline$\hat{C}_{7}$ & Balu & vs & Syngenta & B 178, B 184, B551 e B 761 & vs & $\begin{array}{c}\text { Fort, Garra, Penta, Pointer, } \\
\text { Premium Flex, Speed, Tork e } \\
\text { Valent }\end{array}$ \\
\hline$\hat{C}_{8}$ & Coodetec & vs & Embrapa & $\begin{array}{c}\text { CD 304, CD 306, CD 307, CD } 308 \\
\text { e OCEPAR } 705\end{array}$ & vs & $\begin{array}{c}\text { BRS 2114, BRS 2160, BRS } 3003 \\
\text { e BRS } 3150\end{array}$ \\
\hline$\hat{C}_{9}$ & Coodetec & vs & Syngenta & $\begin{array}{c}\text { CD 304, CD 306, CD 307, CD } 308 \\
\text { e OCEPAR } 705\end{array}$ & vs & $\begin{array}{c}\text { Fort, Garra, Penta, Pointer, } \\
\text { Premium Flex, Speed, Tork e } \\
\text { Valent }\end{array}$ \\
\hline$\hat{C}$ & Embrapa & vs & Syngenta & $\begin{array}{c}\text { BRS 2114, BRS 2160, BRS } 3003 \text { e } \\
\text { BRS } 3150\end{array}$ & vs & $\begin{array}{c}\text { Fort, Garra, Penta, Pointer, } \\
\text { Premium Flex, Speed, Tork e } \\
\text { Valent }\end{array}$ \\
\hline
\end{tabular}

\section{Resultados e Discussão}

Os contrastes realizados entre procedências na dosagem de $30 \mathrm{~g} \mathrm{ha}^{-1}$ de nicosulfuron (Tabela 5) evidenciaram que os híbridos Balu, Coodetec e Embrapa foram os únicos que apresentaram efeitos significativos de redução de massa seca de parte aérea, sendo que as três procedências, em média, foram mais sensíveis que os híbridos Agroceres, Dow, Nidera e Pionner. No entanto, os híbridos Coodetec, em média, foram também mais sensíveis que os híbridos Agroeste, Balu, Guerra e Syngenta, e os híbridos Embrapa, em média, foram mais sensíveis que os híbridos Agroeste e Syngenta.

Tabela 5. Contrastes entre procedências dos híbridos de milho utilizados, estimativas obtidas e probabilidade de significância para o teste $\mathrm{F}$ da variável-resposta produção de massa seca relativa de parte aérea (MSPA), na dosagem de $30 \mathrm{~g} \mathrm{ha}^{-1}$ de nicosulfuron. Maringá-PR, 2005.

\begin{tabular}{|c|c|c|c|c|c|c|c|c|c|}
\hline \multicolumn{2}{|l|}{$\begin{array}{c}\text { Coeficientes } \\
\oplus\end{array}$} & $\begin{array}{c}\text { Coeficientes } \\
\Theta\end{array}$ & $\begin{array}{c}\text { Estimativa } \\
\text { dos } \\
\text { contrastes }\end{array}$ & $\operatorname{Pr}>\mathbf{f}$ & $\begin{array}{c}\text { Coeficientes } \\
\oplus\end{array}$ & & $\begin{array}{c}\text { Coeficientes } \\
\Theta\end{array}$ & $\begin{array}{c}\text { Estimativa } \\
\text { dos } \\
\text { contrastes }\end{array}$ & $\operatorname{Pr}>\mathbf{f}$ \\
\hline \multicolumn{3}{|c|}{ Contrastes entre procedências } & \multicolumn{2}{|c|}{ MSPA } & \multicolumn{3}{|c|}{ Contrastes entre procedências } & \multicolumn{2}{|c|}{ MSPA } \\
\hline Agroceres & $v s$ & Agroeste & $+1,570$ & 0,498 & Balu & $v s$ & Syngenta & $-3,938$ & 0,119 \\
\hline Balu & $v s$ & Agroceres & $-5,284$ & 0,046 & Coodetec & $v s$ & Dow & $-12,282$ & $<0,001$ \\
\hline Coodetec & $v s$ & Agroceres & $-11,327$ & $<0,001$ & Embrapa & $v s$ & Coodetec & $+3,032$ & 0,336 \\
\hline Dow & $v s$ & Agroceres & $+0,956$ & 0,695 & Guerra & $v s$ & Coodetec & $+11,147$ & 0,005 \\
\hline Agroceres & $v s$ & Embrapa & $+8,295$ & 0,006 & Nidera & $v s$ & Coodetec & $+13,981$ & $<0,001$ \\
\hline Agroceres & $v s$ & Guerra & $+0,180$ & 0,963 & Pionner & $v s$ & Coodetec & $+13,403$ & $<0,001$ \\
\hline Nidera & $v S$ & Agroceres & $+2,654$ & 0,314 & Coodetec & $v s$ & Syngenta & $-9,980$ & $<0,001$ \\
\hline Pionner & $v s$ & Agroceres & $+2,076$ & 0,431 & Embrapa & $v s$ & Dow & $-9,251$ & 0,002 \\
\hline Agroceres & $v s$ & Syngenta & $+1,347$ & 0,561 & Guerra & $v s$ & Dow & $-1,136$ & 0,768 \\
\hline Balu & $v s$ & Agroeste & $-3,714$ & 0,141 & Nidera & $v s$ & Dow & $+1,699$ & 0,519 \\
\hline Coodetec & $v s$ & Agroeste & $-9,757$ & $<0,001$ & Dow & $v s$ & Pionner & $-1,120$ & 0,671 \\
\hline Dow & $v s$ & Agroeste & $+2,525$ & 0,276 & Dow & $v s$ & Syngenta & $+2,302$ & 0,320 \\
\hline
\end{tabular}




\begin{tabular}{ccccccccccc} 
Embrapa & $v s$ & Agroeste & $\mathbf{- 6 , 7 2 6}$ & 0,020 & Guerra & $v s$ & Embrapa & $+8,115$ & 0,056 \\
Agroeste & $v s$ & Guerra & $-1,390$ & 0,713 & Embrapa & $v s$ & Nidera & $\mathbf{- 1 0 , 9 4 9}$ & 0,001 \\
Agroeste & $v s$ & Nidera & $-4,224$ & 0,094 & Embrapa & $v s$ & Pionner & $\mathbf{- 1 0 , 3 7 1}$ & 0,001 \\
Agroeste & $v s$ & Pionner & $-3,645$ & 0,149 & Embrapa & $v s$ & Syngenta & $\mathbf{- 6 , 9 4 9}$ & 0,017 \\
Agroeste & $v s$ & Syngenta & $-0,223$ & 0,919 & Guerra & $v s$ & Nidera & $-2,834$ & 0,477 \\
Balu & $v s$ & Coodetec & $\mathbf{+ 6 , 0 4 3}$ & 0,033 & Guerra & $v s$ & Pionner & $-2,556$ & 0,571 \\
Balu & $v s$ & Dow & $\mathbf{- 6 , 2 4 0}$ & 0,018 & Guerra & $v s$ & Syngenta & $+1,167$ & 0,758 \\
Embrapa & $v s$ & Balu & $\mathbf{- 3 , 0 1 1}$ & 0,339 & Pionner & $v s$ & Nidera & $-0,578$ & 0,837 \\
Guerra & $v s$ & Balu & $+5,104$ & 0,201 & Nidera & $v s$ & Syngenta & $+4,001$ & 0,113 \\
Nidera & $v s$ & Balu & $\mathbf{+ 7 , 9 3 8}$ & 0,005 & Pionner & $v s$ & Syngenta & $+3,422$ & 0,175 \\
Pionner & $v s$ & Balu & $\mathbf{+ 7 , 3 6 0}$ & 0,009 & - & - & - & - & - \\
\hline
\end{tabular}

*As estimativas apresentadas em negrito são significativas pelo teste $\mathrm{F}$ a $5 \%$ de probabilidade.

Comparando as diferentes procedências, esses resultados indicam que os híbridos Balu, Coodetec e Embrapa destacam-se como menos tolerantes à dosagem de $30 \mathrm{~g} \mathrm{ha}^{-1}$, especialmente no que se refere aos híbridos Coodetec, que foram significativamente mais sensíveis que todas as demais procedências, com exceção dos híbridos Embrapa.

Com relação aos contrastes realizados entre procedências na dosagem de $60 \mathrm{~g} \mathrm{ha}^{-1}$ de nicosulfuron (Tabelas 6), os híbridos Balu, Coodetec e Syngenta apresentaram efeito significativo de redução de massa seca de parte aérea, sendo os dois primeiros, em média, mais sensíveis que os híbridos Agroceres, Agroeste, Dow, Embrapa, Guerra, Nidera, Pionner e Syngenta, e os híbridos Syngenta, em média, mais sensíveis que os híbridos Agroeste, Dow, Embrapa, Nidera e Pionner. Nesta dosagem, os híbridos Pionner, em média, foram mais tolerantes que as demais procedências, com exceção aos híbridos Embrapa, que, ao contrário do ocorrido na dosagem de $30 \mathrm{~g} \mathrm{ha}^{-1}$, não mostraram sensibilidade inferior a nenhuma das outras procedências, provavelmente devido a um efeito mais acentuado desta dosagem sobre os demais híbridos.

Tabela 6. Contrastes entre procedências dos híbridos de milho utilizados, estimativas obtidas e probabilidade de significância para o teste $\mathrm{F}$ da variável-resposta produção de massa seca relativa de parte aérea (MSPA), na dosagem de $60 \mathrm{~g} \mathrm{ha}^{-1}$ de nicosulfuron. Maringá-PR, 2005.

\begin{tabular}{|c|c|c|c|c|c|c|c|c|c|}
\hline \multicolumn{2}{|l|}{$\begin{array}{c}\text { Coeficientes } \\
\oplus\end{array}$} & $\begin{array}{c}\text { Coeficientes } \\
\Theta\end{array}$ & $\begin{array}{l}\text { Estimativa } \\
\text { dos } \\
\text { contrastes }\end{array}$ & $\operatorname{Pr}>\mathbf{f}$ & \multicolumn{2}{|c|}{$\begin{array}{c}\text { Coeficientes } \\
\oplus\end{array}$} & $\begin{array}{c}\text { Coeficientes } \\
\Theta\end{array}$ & $\begin{array}{l}\text { Estimativa } \\
\text { dos } \\
\text { contrastes }\end{array}$ & $\operatorname{Pr}>\mathbf{f}$ \\
\hline \multicolumn{3}{|c|}{ Contrastes entre procedências } & \multicolumn{2}{|l|}{ MSPA } & \multicolumn{3}{|c|}{ Contrastes entre procedências } & \multicolumn{2}{|l|}{ MSPA } \\
\hline Agroceres & $v s$ & Agroeste & $-2,835$ & 0,221 & Balu & $v s$ & Syngenta & $-7,544$ & 0,003 \\
\hline Balu & $v s$ & Agroceres & $-11,647$ & $<0,001$ & Coodetec & $v s$ & Dow & $-19,785$ & $<0,001$ \\
\hline Coodetec & $v s$ & Agroceres & $-15,552$ & $<0,001$ & Embrapa & $v s$ & Coodetec & $+20,324$ & $<0,001$ \\
\hline Dow & $v s$ & Agroceres & $+4,233$ & 0,083 & Guerra & $v s$ & Coodetec & $+14,217$ & $<0,001$ \\
\hline Agroceres & $v s$ & Embrapa & $-4,773$ & 0,111 & Nidera & $v s$ & Coodetec & $+17,316$ & $<0,001$ \\
\hline Agroceres & $v s$ & Guerra & $+1,335$ & 0,729 & Pionner & $v s$ & Coodetec & $+25,977$ & $<0,001$ \\
\hline Nidera & $v s$ & Agroceres & $+1,764$ & 0,503 & Coodetec & $v s$ & Syngenta & $-11,449$ & $<0,001$ \\
\hline Pionner & $v s$ & Agroceres & $+10,425$ & $<0,001$ & Embrapa & $v s$ & Dow & $+0,539$ & 0,857 \\
\hline Agroceres & $v s$ & Syngenta & $+4,103$ & 0,077 & Guerra & $v s$ & Dow & $-5,568$ & 0,150 \\
\hline Balu & $v s$ & Agroeste & $-14,482$ & $<0,001$ & Nidera & $v s$ & Dow & $-2,470$ & 0,349 \\
\hline
\end{tabular}




\begin{tabular}{|c|c|c|c|c|c|c|c|c|c|}
\hline Coodetec & $v s$ & Agroeste & $-18,387$ & $<0,001$ & Dow & $v s$ & Pionner & $-6,192$ & 0,019 \\
\hline Dow & $v s$ & Agroeste & $+1,398$ & 0,546 & Dow & $v s$ & Syngenta & $+8,336$ & $<0,001$ \\
\hline Embrapa & $v s$ & Agroeste & $+1,938$ & 0,502 & Guerra & $v s$ & Embrapa & $-6,108$ & 0,149 \\
\hline Agroeste & $v s$ & Guerra & $+4,170$ & 0,270 & Embrapa & $v s$ & Nidera & $+3,008$ & 0,340 \\
\hline Agroeste & $v s$ & Nidera & $+1,071$ & 0,671 & Embrapa & $v s$ & Pionner & $-5,653$ & 0,073 \\
\hline Agroeste & $v s$ & Pionner & $-0,590$ & 0,003 & Embrapa & $v s$ & Syngenta & $+8,876$ & 0,002 \\
\hline Agroeste & $v s$ & Syngenta & $+6,938$ & 0,002 & Guerra & $v s$ & Nidera & $-3,099$ & 0,437 \\
\hline Balu & $v s$ & Coodetec & $+3,905$ & 0,166 & Guerra & $v s$ & Pionner & $-11,760$ & 0,003 \\
\hline Balu & $v s$ & Dow & $-15,880$ & $<0,001$ & Guerra & $v s$ & Syngenta & $+2,768$ & 0,464 \\
\hline Embrapa & $v s$ & Balu & $+16,419$ & $<0,001$ & Pionner & $v s$ & Nidera & $+8,661$ & 0,002 \\
\hline Guerra & $v s$ & Balu & $+10,312$ & 0,010 & Nidera & $v s$ & Syngenta & $+5,867$ & 0,020 \\
\hline Nidera & $v s$ & Balu & $+13,411$ & $<0,001$ & Pionner & $v s$ & Syngenta & $+14,528$ & $<0,001$ \\
\hline Pionner & $v s$ & Balu & $+22,072$ & $<0,001$ & - & - & - & - & - \\
\hline
\end{tabular}

* As estimativas apresentadas em negrito são significativas pelo teste $\mathrm{F}$ a $5 \%$ de probabilidade.

Contudo, analisando-se os contrastes realizados nas dosagens de 30 e $60 \mathrm{~g} \mathrm{ha}^{-1}$ de nicosulfuron, podese inferir que os híbridos Balu e Coodetec, de modo geral, podem ser considerados de maior sensibilidade ao herbicida nicosulfuron se comparados aos outros híbridos estudados.

Os contrastes realizados entre procedências na dosagem de $60 \mathrm{~g} \mathrm{ha}^{-1}$ de isoxaflutole (dados não demonstrados) não estabeleceram diferenças significativas de massa seca de parte aérea entre os grupos analisados. Isso pode indicar, de modo geral, uma situação de alta tolerância para as diferentes procedências nesta dosagem do herbicida. Os contrastes realizados na dosagem de $120 \mathrm{~g} \mathrm{ha}^{-1}$ (Tabela 7) somente evidenciaram que os híbridos Balu, em média, apresentaram massa seca de parte aérea significativamente superior aos híbridos Embrapa.

Tabela 7. Contrastes entre procedências dos híbridos de milho utilizados, estimativas obtidas e probabilidade de significância para o teste $\mathrm{F}$ da variável-resposta matéria seca da parte aérea (MSPA), na dosagem de $120 \mathrm{~g} \mathrm{ha}^{-1} \mathrm{de}$ isoxaflutole. Maringá-PR, 2004.

\begin{tabular}{|c|c|c|c|c|c|c|c|c|c|}
\hline \multicolumn{2}{|l|}{$\begin{array}{c}\text { Coeficientes } \\
\oplus\end{array}$} & $\begin{array}{c}\text { Coeficientes } \\
\Theta\end{array}$ & $\begin{array}{c}\text { Estimativ } \\
\text { dos } \\
\text { contraste }\end{array}$ & $\operatorname{Pr}>\mathbf{f}$ & $\begin{array}{c}\text { Coeficiente } \\
\oplus\end{array}$ & & $\begin{array}{c}\text { Coeficientes } \\
\Theta\end{array}$ & $\begin{array}{c}\text { Estimativa } \\
\text { dos } \\
\text { contrastes }\end{array}$ & $\operatorname{Pr}>\mathbf{f}$ \\
\hline \multicolumn{3}{|c|}{ Contrastes entre procedências } & \multicolumn{2}{|c|}{ MSPA } & \multicolumn{3}{|c|}{ Contrastes } & \multicolumn{2}{|c|}{ MSPA } \\
\hline Agroceres & vs & Balu & $-2,973$ & 0,602 & Balu & vs & Embrapa & $+10,425$ & 0,026 \\
\hline Agroceres & vs & Coodetec & $+4,336$ & 0,431 & Balu & vs & Syngenta & $+3,216$ & 0,425 \\
\hline Agroceres & vs & Embrapa & $+7,453$ & 0,192 & Embrapa & vS & Coodetec & $-3,117$ & 0,481 \\
\hline Agroceres & vs & Syngenta & $+0,243$ & 0,963 & Coodetec & vs & Syngenta & $-4,093$ & 0,276 \\
\hline Balu & vs & Coodetec & $+7,309$ & 0,099 & Embrapa & vs & Syngenta & $-7,209$ & 0,075 \\
\hline
\end{tabular}

* As estimativas apresentadas em negrito são significativas pelo teste $\mathrm{F}$ a $5 \%$ de probabilidade. 
Segundo Van Eerd et al. (2003), alguns agroquímicos podem estimular ou prejudicar a ação do complexo enzimático P-450, que é responsável por uma das fases de metabolismo de herbicidas em plantas, fato que pode afetar seu crescimento e desenvolvimento.

Algumas explicações podem esclarecer os possíveis mecanismos de tolerância de híbridos de milho aos herbicidas nicosulfuron e isoxaflutole, entre elas, sugere-se a maior ou menor atividade de enzimas monoxigenases dependentes do citocromo P-450, glutationa S-transferase (GSTs), esterases e UDP-glicosiltransferases, responsáveis pela desintoxicação de herbicidas (FERREIRA; CATANEO, 2001). Outras explicações sugerem a absorção e translocação diferencial do herbicida para o sítio de ação na planta, com maior ou menor capacidade de metabolização e desintoxicação (EZRA; GRASSEL, 1982).

No caso das sulfoniluréias, a sua adoção requer a observação de alguns fatores além do híbrido utilizado, tais como estádio fenológico da cultura no instante da aplicação e o intervalo entre a aplicação do herbicida e inseticida organofosforado ou mesmo da adubação nitrogenada de cobertura, que, quando negligenciados, podem interferir em sua seletividade e causar fitointoxicação à cultura (LÓPEZ OVEJERO et al., 2003).

Em relação ao isoxaflutole, acredita-se que da mesma forma como ocorre em espécies sensíveis, um híbrido de milho pode ser mais sensível que outro em função da metabolização lenta da molécula, permitindo a inibição enzimática pelo diquetonitrila que é a molécula ativa.

Contudo, tem-se como hipótese que a tolerância entre procedências de híbridos de milho para ambos os herbicidas pode ser justificada pela maior ou menor capacidade dos híbridos de absorver, translocar e metabolizar as moléculas estudadas. Concluiu-se que existe diferença de tolerância entre procedências de híbridos de milho em relação à suscetibilidade aos herbicidas nicosulfuron $\mathrm{e}$ isoxaflutole. Em média, as procedências Balu e Coodetec foram mais sensíveis ao nicosulfuron do que as demais procedências. Na dosagem de $120 \mathrm{~g}$ $\mathrm{ha}^{-1}$ de isoxaflutole, em média, a procedência Balu mostrou-se mais tolerante que a Embrapa.

\section{Agradecimentos}

Os autores agradecem ao Conselho Nacional de Desenvolvimento Científico (CNPq) pela bolsa de mestrado concedida ao primeiro autor e aos funcionários do Núcleo de Estudos Avançados em Ciência das Plantas Daninhas Luiz Machado Homem e Milton Lopes da Silva, pelo auxílio prestado durante a condução dos experimentos.

\section{Referências}

ANDERSON, D. D.; NISSEN, S. J.; MARTIN, A. R.; ROETH, F. W. Mechanism of primisulfuron resistance in a shattercane (Sorghum bicolor) biotype. Weed Science, Lawrence, v. 46, n. 1, p. 158-162, 1998.

BROW, H. M.; DIETRICH, R. F.; KENYON, W. H.; LICHTNER, F. T. Prospects for the biorational design of crop selective herbicides. Brighton Crop Protection Conference-Weeds, Switzerland, v. 7, n. 2, p. 847-856, 1991.

CAREY, J. B.; PENNER, D.; KELLS, J. J. Physiological basis for nicosulfuron and primisulfuron selectivity in five plant species. Weed Science, Lawrence, v. 45, n. 1, p. 22-30, 1997.

CEZARINO, V. Isoxaflutole - nova molécula herbicida para as culturas da cana-de-açúcar e do milho. In: CONGRESSO BRASILEIRO DA CIÊNCIA DAS PLANTAS DANINHAS, 21., 1997, Caxambu. Palestras... Caxambu: SBCPD, 1997. p. 79-93.

EMPRESA BRASILEIRA DE PESQUISA AGROPECUÁRIA - EMBRAPA. Sistema brasileiro de classificação de solos. Rio de Janeiro: Embrapa Solos, 1999.

EZRA, G.; GRESSEL, J. Rapid effects of a thiocarbamate herbicide and its dichloroacetamide protectant on a macromolecular synthesis and glutathione levels in maize cell cultures. Pesticide Biochemistry Physiology, Duluth, v. 17, n. 1, p. 48-58, 1982. 
FERREIRA, D. F. Sistema de análise de variância (Sisvar): versão 4.6. Lavras: Departamento de Ciências Exatas da UFLA, 1999.

FERREIRA, L. C.; CATANEO, A. C. Aspectos bioquímicos da ação "de safeners". Boletim Informativo da SBCPD, São Paulo, v. 8, n. 2, p. 5-6, 2001.

FONNE-PFISTER, R.; GAUDIN, J.; KREUZ, K.; RAMSTEINER, K.; EBERT, E. Hydroxylation of primisulfuron by an inducible cytochrome P450dependent monooxygenase system from maize. Pesticide Biochemistry Physiology, Duluth, v. 37, n. 1, p. 165-173, 1990.

GUBBigA, N. G.; WORShAM, A. D.; COBLE, H. D. Effect of nicosulfuron on johnsongrass (Sorghum halepense) control and corn (Zea mays) performance. Weed Technology, Champaign, v. 9, n. 3, p. 3574-3581, 1995.

HARMS, C. T.; MONTOYA, A. L.; PRIVALLE, L. S.; BRIGGS, R. W. Genetic and biochemical characterization of corn inbred lines tolerant to the sulfonylurea primisulfuron. Theoretical and Applied Genetics, Berlin, v. 80, n. 3 , p. $353-358,1990$.

LÓPEZ-OVEJERO, R. F.; CHRISTOFFOLETI, P. J.; NICOLAI, M.; BARELA, J. F. Manejo de plantas daninhas na cultura do milho. In: FANCELLI, A. L.; DOURADO NETO, D. (Ed.). Milho: estratégias de manejo para alta produtividade. Piracicaba: Escola Superior de Agricultura "Luiz de Queiroz", 2003. p. 47-79.

PALlET, K. E.; LITTLE, J. P.; SHEEKEY, M.; VEERASEKARAN, P. The mode of action of isoxaflutole. I. Physiological effects, metabolism, and selectivity. Pesticide Biochemistry Physiology, Duluth, v. 62, n. 2, p. 113-124, 1998.

RODRIGUES, B. N.; ALMEIDA, F. S. Guia de herbicidas. 5. ed. Londrina: Ed. dos Autores, 2005.

SPRAGUE, C. L.; PENNER, D.; KELLS, J. J. Physiological basis for tolerance of four Zea mays hybrids to RPA 201772. Weed Science, Lawrence, v. 47, n. 4, p. 375-382, 1999.

STATISTICAL ANALYSIS SYSTEM INSTITUTE SAS. Procedure guide for personal computers: Version 5. Cary: SAS Inst, 1999.

VANEERD,L.L.;HOAGLAND,R.E.;ZABLOTOWICZ, R. M.; HALL, J. C. Pesticide metabolism in plants and microorganisms. Weed Science, Lawrence, v. 51, n. 4, p. 472-495, 2003.

VIVIANI, F.; LITTLE, J. P.; PALLET, K. E. The mode of action of isoxaflutole. II. Characterization of the inhibition of carrot 4-hydroxyphenylpyruvate dioxygenase by the diketonitrile derivative of isoxaflutole. Pesticide Biochemistry Physiology, Duluth, v. 62, n. 2, p. 125-134, 1998. 
